www.jmscr.igmpublication.org

Impact Factor (SJIF): 6.379

Index Copernicus Value: 71.58

ISSN (e)-2347-176x ISSN (p) 2455-0450

crossref DOI: _https://dx.doi.org/10.18535/jmscr/v6i3.154

Journal Of Medical Science And Clinical Research

IGM Publication

An Official Publication of IGM Publication

\title{
Intraoperative Findings in Patients of Peptic Ulcer Perforation: A Prospective Study in Correlation with Patient Outcome
}

\author{
Authors \\ Dr Vinod Yedalwar ${ }^{1}$, Dr Rajeev Kaneria ${ }^{2}$, Dr Atul Kumar Singh ${ }^{3}$, Dr Lalmani Singh ${ }^{4}$ \\ ${ }^{1,3,4}$ Associate Professor, Department of Surgery, Shyam Shah Medical College, Rewa, MP \\ ${ }^{2}$ Junior Resident, Department of Surgery, Shyam Shah Medical College, Rewa, MP \\ Corresponding Author \\ Dr Rajeev Kaneria
}

Address: 186, Sai Kripa Colony, Indore (M.P.) India

Email: rajeevkaneria2701@ gmail.com, Phone Number: 7000046849

\begin{abstract}
Background: Peptic ulcer perforation is an emergency and requires prompt diagnosis and urgent surgical intervention. Overall, peptic ulcer mortality and hospitalization rates have declined for the past two decades, but complications such as peptic ulcer perforation and bleeding remain a substantial health-care problem.

Material and Methods: A prospective 1-year study conducted in 116 cases of peptic perforation admitted in surgical wards of Sanjay Gandhi Memorial Hospital, Rewa M.P. during the study period June 2016-May 2017.All patients suspected of peptic perforation were admitted to surgery ward and underwent emergency exploratory laparotomy. Post-operatively $H$. pylori serological test to detect IgG antibody was done with patients blood serum. Their findings were recorded in a proforma and master chart. The information obtained was tabulated and analyzed.

Results: Incidence of peptic perforation was 116 (1.20\%) cases of all surgical admissions, Most common site of peptic perforation was anterior wall of first part of duodenum in 61 (55.4\%) cases, omentopexy was most common procedure done i.e. in 106 (91.37\%) cases, overall Mortality was 15 (12.93\%) cases.

Conclusion: Perforated peptic ulcer is a disease of middle aged adults. Delay in hospitalization due to initial treatment by homemade medicines and non availability of essential surgical care further complicates the perforation in this region.

Perforation is diagnosed on clinical grounds immediately as patient reaches emergency department yet due to delayed hospitalization, time lost in resuscitation of the patient affects the outcome of standard surgical procedure. Selection of appropriate surgical procedure and postoperative care is helpful in early and uneventful recovery. Post operative H. pylori eradication therapy follow up endoscopic facilities in patients found positive for $H$. pylori can reduce recurrence rates and subsequently the burden of this disease.

Keywords: Peptic ulcer perforation, intraoperative findings, Outcome.
\end{abstract}

\section{Introduction}

Peptic ulcer perforation is a common life threatening emergency and requires urgent surgical intervention. ${ }^{1}$ Although the frequency of ulcer disease in general has declined, the number of patients affected by bleeding and perforation 
has not changed significantly. The sudden release of gastric or duodenal contents into the peritoneal cavity through a perforation leads to a devastating sequence of events which if not properly managed, is likely to cause death. Studies have suggested that if signs of peritonitis are present the exploratory laparotomy should be done. ${ }^{2}$ This should be done within 12 hours to avoid poor outcome. ${ }^{3}$ Various surgical options are available and choice depends on duration of peritonitis, size of perforation, past history of symptomatic peptic ulcer disease and co-morbid conditions. ${ }^{4}$ Prompt recognition of the condition is very important and only by early diagnosis and treatment it is possible to reduce the still relatively high mortality.

\section{Material and Methods}

The present study "Intraoperative Findings in Patients of Peptic Ulcer Perforation: A Prospective Study in Corelation with Patient Outcome" was carried out in 116 cases admitted in Surgical wards of Department of Surgery, Shyam Shah Medical College and associated Sanjay Gandhi Memorial Hospital Rewa during $1^{\text {st }}$ June 2016 to $31^{\text {st }}$ May 2017.

Patients of peptic perforation were admitted through casualty department, surgical OPD or transferred from other departments. Patients were diagnosed on clinical grounds with classical symptoms like epigastric pain, distension, vomiting, fever, not passing flatus and stools and not passing urine. Patients examined for signs of shock, dehydration. General examination was done for vital status, pallor, jaundice, edema, nutritional status and abdomen was examined for distension, tenderness, rigidity \& guarding, obliteration of liver dullness, free fluid, absence of bowel sounds. Also complete personal history was obtained which includes smoking, alcoholism, tobacco chewing, analgesic abuse and history of other comorbidities. All findings were recorded in details.

Investigations like complete blood counts, LFT, RFT, Random Blood sugar, Coagulation Profile,
Blood Grouping, Serum electrolytes, X-ray chest and abdomen erect view was done. After resuscitation and stabilization of vitals, patient is prepared for surgery- Exploratory laparotomy was done to deal with pathology. Post-operatively $\mathrm{H}$. pylori serological test to detect IgG antibody was done with patients blood serum. Patients who tested positive for $\mathrm{H}$. pylori were given Anti $\mathrm{H}$. Pylori Drug Therapy and those who tested negative were advised to take PPIs. Patients managed in wards post-operatively. Regular follow up done in SOPD.

All these data was recorded meticulously in proforma and in master chart for further systematic tabulation for observations and analysis.

\section{Results}

Total 116 patients of perforation were included in this study out of which 110 patient underwent exploratory laparotomy. Incidence of peptic perforation was $1.20 \%$ of all surgical admissions. Maximum number of peptic perforation cases were found in age group31-40 years $(20.68 \%)$. Peptic perforation was more common in male than female patients and male to female ratio was 13.5:1. Most common site of peptic perforation was anterior wall of first part of duodenum in 61 $(55.4 \%)$ cases, followed by prepyloric region of stomach $47(42.72 \%)$ cases and in body of stomach in $2(1.81 \%)$ cases. The average size of perforation, in $94(85.4 \%)$ cases was less than 1 $\mathrm{cm}, 1-3 \mathrm{~cm}$ in $14(12.72)$ cases and $>3 \mathrm{~cm}$ in 2 $(1.81 \%)$ cases. Nature of peritoneal fluid was bilious in $92(83.6 \%)$ cases, bilious + pus in $12(11 \%)$ cases and pus in $6(5.4 \%)$ cases.

(Table-1). Serology for $H$. pylori infection was positive in 25 cases $(41 \%)$ of duodenal perforation and in 11 cases (22.44\%) of prepyloric perforation. Among $H$. pylori positive cases, in $69.4 \%$ cases site of perforation was first part of duodenum, and in $30.6 \%$ site of perforation was prepyloric area of stomach (Table- 2). 
Table-1 Distribution of cases according to Intra Operative Findings

\begin{tabular}{|l|c|c|c|}
\hline S. No. & Intra operative findings & No. of cases & Percentage(\%) \\
\hline 1 & \multicolumn{3}{|c|}{ Site of perforation } \\
\hline & $1^{\text {st }}$ part of Duodenum & 61 & 55.4 \\
\hline & Prepyloric region & 47 & 42.72 \\
\hline & Body of Stomach & 2 & 1.81 \\
\hline 2 & \multicolumn{3}{|c|}{ Size of perforation } \\
\hline & Upto $1 \mathrm{~cm}$ & 94 & 85.4 \\
\hline & $1-3 \mathrm{~cm}$ & 14 & 12.72 \\
\hline & $>3 \mathrm{~cm}$ & 2 & 1.81 \\
\hline 3 & \multicolumn{3}{|c|}{ Peritoneal Fluid } \\
\hline & Bilious & 92 & 83.6 \\
\hline & Pus & 6 & 5.4 \\
\hline & Bilious+ Pus & 12 & 11 \\
\hline
\end{tabular}

Table -2 Incidence of $H$. pylori positive cases in peptic perforation according to site of perforation

\begin{tabular}{|l|l|c|c|c|}
\hline S. No. & Site of Perforation & Total Cases & No. of positive cases & Percentage(\%) \\
\hline 1 & First part of Duodenum & 61 & 25 & $41 \%$ \\
\hline 2 & Prepyloric area of stomach & 49 & 11 & $22.44 \%$ \\
\hline
\end{tabular}

In present series, 110 cases underwent surgery out of which omentopexy was done in 106 (91.37\%) cases, omentopexy with gastrojejunostomy was done in 3 cases $(2.58 \%)$ and omentopexy with feeding jejunostomy was done in 1 case.

Table-3 Distribution of cases according to Postoperative complications

\begin{tabular}{|l|c|c|c|}
\hline \multirow{2}{*}{ S. No. } & Complication & \multicolumn{2}{|c|}{ Cases of Peptic Perforation } \\
\cline { 3 - 4 } & & No. of cases & Percentage(\%) \\
\hline 1 & Pulmonary & 35 & 31.8 \\
\hline 2 & Toxaemia & 13 & 11.8 \\
\hline 3 & Renal & 5 & 4.5 \\
\hline 4 & Cardiovascular & 9 & 8.2 \\
\hline 5 & Neurological & 5 & 4.5 \\
\hline 6 & Surgical Site & 46 & 41.8 \\
\hline \multirow{3}{*}{} & Wound infection & 13 & 11.8 \\
\cline { 2 - 4 } & Wound Dehiscence & 4 & 3.6 \\
\cline { 2 - 4 } & Burst Abdomen & 3 & 2.72 \\
\cline { 2 - 4 } & Biliary leak & 9 & 8.2 \\
\hline 7 & None & & \\
\hline
\end{tabular}

Intraperitoneal contamination was more in patients presented to hospital after 72 hours of onset, these patients also had more postoperative complications and more mortality.

Biliary leak seen in 3 cases, out of which Reoperation done in 2 cases. Among 3 cases:-

One patient presented with biliary leak on 3rd postoperative day, patient was in septicaemic shock, his general condition was poor and was not a not suitable candidate for re-exploration, patient expired on postoperative day 5 .

In other 2 patients, re-exploration was done. In one case, primary closure of peptic perforation
The commonest post operative complication was wound infection (41.8\%) followed by Pulmonary complications $(31.8 \%)$ (Table -3$)$. 
Table- 4 Mortality of cases in relation to duration of onset of symptoms to presentation at hospital

\begin{tabular}{|l|c|c|c|c|}
\hline S. No. & Duration & Total cases & Total Death & Percentage(\%) \\
\hline 1 & $<24$ hours & 21 & 2 & 9.52 \\
\hline 2 & $24-72$ hours & 53 & 5 & 9.43 \\
\hline 3 & $>72$ hours & 42 & 8 & 19.04 \\
\hline & Total & 116 & 15 & 12.93 \\
\hline
\end{tabular}

Average hospital stay was maximum (22 days) in patients treated by omentopexy with Gastrojejunostomy with feeding jejunostomy. Average hospital stay was 6.8 days in patients treated conservatively and about 10.6 days in patients treated with omentopexy. Overall average duration of stay was $10.54 \pm 5.90$ days (Table-5).

Table-5 Distribution of cases according to Hospital Stay

\begin{tabular}{|l|c|c|c|}
\hline S. No. & Procedure done & No. of cases & Avg. Stay(in days) \\
\hline 1 & Conservative & 6 & 6.8 \\
\hline 2 & Operative & 110 & 10.5 \\
\hline \multirow{3}{*}{} & Intraperitoneal drainage followed by Exp. Lap. & 13 & 15.1 \\
\cline { 2 - 4 } & Omentopexy & 106 & 10.6 \\
\cline { 2 - 4 } & omentopexy with Gastrojejunostomy & 3 & 14 \\
\cline { 2 - 4 } & $\begin{array}{c}\text { omentopexy with Gastrojejunostomy with } \\
\text { feeding jejunostomy }\end{array}$ & 1 & 22 \\
\hline
\end{tabular}

\section{Discussion}

Perforation peritonitis is a frequently encountered surgical emergency. Perforated peptic ulcer is a serious complication of PUD with potential risk of grave complications.

Incidence of peptic perforation was $1.20 \%$ of all surgical admissions. Dakubo (2009) reported an incidence of $1.6 \%$ of peptic ulcer perforation. ${ }^{5}$ Maximum number of peptic perforation cases were found in age group31-40 years(20.68\%). Analgesic abuse and addiction to tobacco was responsible for high incidence in this age group. Mean age of presentation was $45.81 \pm 16.17$ years. This is similar to that of Parameshwara Chaldiganahalli Munikrishna (2016). ${ }^{6}$ A M AlMarsoumi (2012) reported peak incidence in 3150 age group. ${ }^{7}$ Peptic perforation was more common in male than female patients and male to female ratio was 13.5:1. A possible explanation for these findings may be that some behaviors, such as Tobacco chewing, smoking and drinking alcohol, are more frequent among men, thus increasing the risk of PUD and perforation, especially in young adults.

Perforation site in $55.4 \%$ cases was anterior wall of $1^{\text {st }}$ part of duodenum and in $42.72 \%$ cases the site of perforation was prepyloric region of stomach and in 2 cases $(1.81 \%)$ perforation was found in body of stomach. The perforation site usually involves the anterior wall of the duodenum (60\%), although it might occur in antral $(20 \%)$ and lesser-curvature gastric ulcers $(20 \%) .{ }^{8}$ Among H. pylori positive cases, in $69.4 \%$ cases site of perforation was first part of duodenum, and in $30.6 \%$ site of perforation was prepyloric area of stomach.

Timely diagnosis of peptic ulcer with detection and eradication of $H$. pylori can reduce unnecessary burden on health infrastructure and its life threatening complications and subsequent mortality and can improve the quality of life for thousands of patients.

It is well known that the bigger the size of perforation the more likely is the development of complications due to increased peritoneal contamination. ${ }^{9}$ The presence of pus in the peritoneal cavity indicates late presentation and bacterial peritonitis with higher risks of complications. In present series, $85.4 \%$ cases the site of perforation was less than or equal to $1 \mathrm{~cm}$ in diameter and in $12.72 \%$ cases the size of perforation was between $1 \mathrm{~cm}$ to $3 \mathrm{~cm}$, in 2 cases 
$(1.81 \%)$ the perforation size was $>3 \mathrm{~cm}$. The nature of intraperitoneal fluid was bilious in $83.6 \%$ cases, pus mixed with bile in $11 \%$ cases.

110 out of 116 cases underwent exploratory laparotomy, it includes 15 cases which were treated initially by peritoneal drainage. In 106 cases Omentopexy was done $(91.37 \%)$, of which 11 cases $(9.48 \%)$ expired. Also 3 cases underwent omentopexy with gastrojejunostomy and Feeding jejunostomy was done in 1 case. Patients in whom perforation size was $<1 \mathrm{~cm}$, general condition was fair, omentopexy was done. In patients who had larger perforation, poor general condition, omentopexy with Gastrojejunostomy and omentopexy with Feeding jejunostomy was done. Duodenal ulcer perforations less than $1 \mathrm{~cm}$ in greatest diameter, and as such, are amenable to closure by omentopexy. ${ }^{10}$ Operative procedures were chosen on the basis of perforation size, peritoneal contamination and fragility of gut.

In present series, wound infection was the most common complication (41.8\%), followed by pulmonary complications $(31.8 \%)$, wound dehiscence in 13 cases $(11.8 \%)$ and burst abdomen in 4 cases(3.6\%). pulmonary complications are due to delayed mobilization, whereas Gross intraperitoneal contamination , poor nutrition and anaemia are responsible for wound infection, wound dehiscence and burst abdomen. Biliary leak seen in 3 cases. Chalya PL (2011), study has shown the commonest postoperative complications were surgical site infections (48\%) and pneumonia (28\%). ${ }^{11}$

Many patients $(36.2 \%)$ presented late to the hospital i.e. more than 72 hours after the onset of symptoms. This is attributed to poverty and lack of awareness of the disease by the patient and relatives and poor index of suspicion by some managing clinicians.

In present study overall mortality was $12.93 \%$ of cases of peptic perforation. Mortality was highest (24\%) in patients of 61-80 years of age which is similar to Chalya 2011 and V Srinivas Goud (2016) as patients in this age group have poor nutritional status and associated comorbidities. ${ }^{11,12}$
In present series, 13 out of 15 deaths observed in cases which presented $>24$ hours after onset of the symptoms as postoperative complications are more in patients presenting late to the hospital.

Average hospital stay in patients with omentopexy was 10.5 days. Average hospital stay was maximum in patient treated by omentopexy with gastrojejunostomy with feeding jejunostomy (22days). Average hospital stay was 15.1 days in cases treated by peritoneal drainage followed by exploratory laparotomy. This is due to the fact that these patients were initially having poor general condition and operated after improvement of general condition and also these patients generally developed wound infection postoperatively, prolonging the hospital stay.

In the present series, it was observed that omentopexy gives better results as compared to other procedures in terms of hospital stay and mortality. As most of these patients were presented early and there was less contamination found intraoperatively which is a major factor in postoperative recovery.

\section{Conclusion}

Perforated peptic ulcer is a disease of middle aged adults. More commonly affects males than females. Tobacco chewing, smoking, alcohol, inadvertent use of analgesics and $H$. pylori infection are most common predisposing factors for peptic ulcer disease and patients inability to get proper and complete treatment is responsible for peptic perforation. Delay in hospitalization due to initial treatment by homemade medicines and non availability of essential surgical care further complicates the perforation in this region.

Perforation is diagnosed on clinical grounds immediately as patient reaches emergency department yet due to delayed hospitalization, time lost in resuscitation of the patient affects the outcome of standard surgical procedure. Selection of appropriate surgical procedure and postoperative care is helpful in early and uneventful recovery. Early diagnosis of peptic ulcer disease and detection of $H$. pylori infection, 
prompt eradication therapy and avoidance of various predisposing factors can help to reduce the morbidity and mortality associated with this global disease.

Post operative $H$. pylori eradication therapy follow up endoscopic facilities in patients found positive for $H$. pylori can reduce recurrence rates and subsequently the burden of this disease.

\section{References}

1. Nuhu A, Madziga AG, Gali BM. Acute perforated duodenal ulcer in Maiduguri. Internet J Surg. 2009;21:1.

2. Testini M, Portincasa $P$, Piccinni G, Lissidini G, Pellegrini F, Greco L. Significant factors associated with fatal outcome in emergency open surgery for perforated peptic ulcer. World $\mathbf{J}$ Gastroenterol. 2003;9:2338-40.

3. Syanes C, Lie RT, Syanes K, Kie SA, Soreide O. Adverse effects of delayed treatment for perforated peptic ulcer. Ann Surg. 1994;220(2):168-75.

4. Hill AG. The management of perforated peptic ulcer in a resource poor environment. East Afr Med J. 2001;78 (8):346-8.

5. Dakubo JC, Naaeder SB, Clegg-Lamptey JN. Gastro-duodenal peptic ulcer perforation. East Afr Med J. 2009; 86(3):100-9.

6. Munikrishna PC, Bojegowda K. A retrospective study on duodenal ulcer perforation and outcome. J. Evid. Based Med. Healthc. 2016; 3(18), 718-722.

7. Al-Marsoumi AM, Jabbo NS. Risk factors in perforated peptic ulcer disease: Incidence and relation to morbidity and mortality. Mustansiriya Med J 2013;12:3544.

8. Zittel TT, Jehle EC, Becker HD: Surgical management of peptic ulcer disease today: indication, technique and outcome. Langenbecks Arch Surg 2000;385:84-96.
9. Kevin GB, and Anthony EY. Peptic ulcer. In: 'The New Aird's Companion in Surgical Studies, 2nd Ed. London: Copyright Licensing Agency Ltd, 90 Nottingham Court Road 1988: 771-72.

10. Chaudhary A, Bose SM, Gupta NM, Wig JD, Khanna SK. Giant Perforations of Duodenal Ulcer. Ind J Gastroenterol 1991;10:14-5.

11. Chalya P, Mabula JB, Koy M, Mchembe MD, Jaka HM, Kabangila R, et al. Clinical profile and outcome of surgical treatment of perforated peptic ulcers in Northwestern Tanzania: A tertiary hospital experience, World J EmergSurg 2011;6:31.

12. Goud VS, Babu NV, Kumar PB. Comparative Study of Closure of Duodenal Perforations with Omental Plugging Versus Graham's Patch. Int J Sci Stud 2016;4(8):138-142. 